\title{
RECOVERY OF MICROSTRUCTURE PROPERTIES: RANDOM VARIABILITY OF SOIL SOLID THERMAL CONDUCTIVITY
}

\author{
DAMIAN STEFANIUK, ADRIAN RÓŻAŃSKI, DARIUSZ ŁYDŻBA \\ Wrocław University of Science and Technology, Faculty of Civil Engineering, Wrocław, Poland, \\ e-mail: adrian.rozanski@pwr.edu.pl (corresponding author)
}

\begin{abstract}
In this work, the complex microstructure of the soil solid, at the microscale, is modeled by prescribing the spatial variability of thermal conductivity coefficient to distinct soil separates. We postulate that the variation of thermal conductivity coefficient of each soil separate can be characterized by some probability density functions: $f_{C l}(\lambda), f_{S i}(\lambda), f_{S a}(\lambda)$, for clay, silt and sand separates, respectively. The main goal of the work is to recover/identify these functions with the use of back analysis based on both computational micromechanics and simulated annealing approaches. In other words, the following inverse problem is solved: given the measured overall thermal conductivities of composite soil find the probability density function $f(\lambda)$ for each soil separate. For that purpose, measured thermal conductivities of 32 soils (of various fabric compositions) at saturation are used. Recovered functions $f(\lambda)$ are then applied to the computational micromechanics approach; predicted conductivities are in a good agreement with laboratory results.
\end{abstract}

Key words: simulated annealing, heat transfer, homogenization, saturated soil

\section{INTRODUCTION}

Soil or rock thermal properties are recently of primary importance in many engineering projects, e.g., in design of geothermal heating and cooling systems, pipelines, buildings in cold regions, underground power cables, in analysis of the earth-sheltered buildings energy demand or the problem of coal gasification, etc. The most desirable thermal parameter is the conductivity $\lambda$ which, in the case of soils, is mainly governed by the conductivity of solid phase $\lambda_{s}$ and the amount of water occupying pore space. Since $\lambda_{s}$ is a non-measurable parameter, therefore in practice, its value is usually postulated ad hoc, without any scientifically based considerations (Gemant, 1952; Johansen, 1975; Farouki, 1981; Côté and Konrad 2005a; Lu et al., 2007).

In this work, we consider the solid phase as a mixture of distinct solid phases, each with prescribed variability of thermal conductivity coefficient. We postulate that thermal conductivity coefficient of each soil separate, i.e., clay, silt or sand can be characterized by the proper probability density function $f(\lambda)$. Since we do not assume the type of the probability distribution, the main goal of the paper is to recover/identify optimum probability density functions $f(\lambda)$. Roughly speaking, we aim to solve the inverse problem: given the set of measured thermal conductivities of soils at saturation, find the probability density functions characterizing the variability of thermal conductivity coefficient of the solid phase. For that purpose we use the back analysis algorithm based on both computational micromechanics and simulated annealing approaches.

The paper is organized as follows. In Section 2, we formulate the problem that is going to be solved within this study. Section 3 deals with the computational micromechanics approach used for evaluation of the homogenized thermal conductivity. The numerical procedure utilized for the recovery of probability density functions is described in Section 4. Next, the results and wide discussion of performed analyses are provided. Final conclusions end the paper.

\section{FORMULATION OF THE PROBLEM}

The overall soil thermal conductivity $\lambda$ is strongly affected by the soil physical properties like poros- 
ity, water content, dry density, etc. The values of these parameters can be easily evaluated by laboratory measurements. The only quantity that cannot be measured directly, but also influencing $\lambda$, is the thermal conductivity of solid phase $\lambda_{s}$. So, its value has to be estimated based on semi-theoretical/ empirical relations (cf. Gemant, 1952; Johansen, 1975; Farouki, 1981; Lu et al., 2007). It is however evident that when the mineral composition of the soil is well recognized and, in addition, if the thermal conductivities of particular minerals are known, then $\lambda_{s}$ can be most accurately estimated by the geometric mean equation (e.g., Cote and Konrad, 2005)

$$
\lambda_{s}=\prod_{i} \lambda_{m_{i}}^{\psi_{i}}
$$

where $\lambda_{m}$ and $\psi$ are the thermal conductivity and the volume fraction of particle-forming mineral $i$. Such approach has, however, certain disadvantages. First, the recognition of the quantitative soil mineralogy is not commonly conducted in engineering practice; this is due to fact that investigation of soil mineral composition is a time consuming and cumbersome procedure. Second difficulty in estimation of $\lambda_{s}$, using equation (1), arises from the fact that even the same minerals can have their own unique internal structure resulting in different values of thermal conductivity, e.g., as reported by Clauser and Huenges (1995), thermal conductivity of quartz can vary from 6 up to $11 \mathrm{~W} \mathrm{~m}^{-1} \mathrm{~K}^{-1}$. The above supports the idea of modelling the soil solid conductivity in the probabilistic sense.

Since each soil separate consists of different minerals (e.g., sand - quartz, clay - illite, kaolinite, etc.), therefore, in the present study we associate the mineral composition of the soil with the soil texture governed by the three distinct fractions: sand, silt and clay. As a consequence, the variation of the conductivity of particular minerals is implied to the appropriate soil separates. In other words, the complex structure of the soil solid, at the mineralogical scale, is modelled by considering the spatial variability of the thermal conductivity with respect to the soil texture. Hence, we assume that the thermal conductivity of each soil separate " $i$ " (clay, silt or sand) can be expressed by a certain probability density function (pdf), say $f_{i}(\lambda)$. Then, if the information on the volumetric fraction of particulate soil separates is available, the variation of the thermal conductivity of solid phase can be described by the following probability density function

$$
f(\lambda)=\phi_{C l} \cdot f_{C l}(\lambda)+\phi_{S i}(\lambda)+\phi_{S a} \cdot \lambda_{S a}(\lambda)
$$

where $\phi$ is the volumetric fraction of soil separate; note that subscripts $\mathrm{Cl}, \mathrm{Si}$ and $\mathrm{Sa}$ denote the soil separates, i.e., clay, silt and sand, respectively.

In this work, we aim to recover (identify) the pdfs $f_{i}(\lambda)$ with the use of micromechanical back analysis. Roughly speaking, it consists in finding the most optimal functions $f_{i}(\lambda)$ ensuring best agreement with laboratory measurements, namely thermal conductivities of soils at saturated state $\lambda^{\text {sat }}$. The details of computational micromechanics approach are provided in the next Section. As a procedure of the back analysis we use the stochastic optimization method, namely the simulated annealing approach (Kirkpatrick et al., 1983). Overall description of the recovery procedure is provided in Section 4.

\section{FRAMEWORK OF THE COMPUTATIONAL MICROMECHANICS}

Within the computational micromechanics approach it is required to specify the geometry of the homogenization domain. Assuming that particular pdfs $f_{i}(\lambda)$ are known, a procedure of $3 \mathrm{D}$ microstructure geometry generation is as follows. First, generate a cubic sample consisting of a very large number of voxels such that each voxel, assigned to the soil separate, is associated with the value of thermal conductivity randomly drawn from the appropriate soil texture pdf (equation (2)). The first part of the procedure ends when the number of randomly occupied voxels is equal to $1-\phi_{w}-\phi_{o m}$; where $\phi_{w}$ and $\phi_{o m}$ are the soil porosity (for fully saturated soil, it is also the content of water) and the organic matter content, respectively. Next, all remaining voxels are randomly prescribed to deterministic (non-random) values of the thermal conductivities of organic matter $\left(\lambda_{o m}=0.25 \mathrm{~W} \mathrm{~m}^{-1} \mathrm{~K}^{-1}\right.$ (Bristow, 2002)) and water $\left(\lambda_{w}=0.6 \mathrm{~W} \mathrm{~m}^{-1} \mathrm{~K}^{-1}\right)$. Obviously, the number of voxels corresponding to organic matter and water phase is equated to $\phi_{o m}$ and $\phi_{w}$, respectively. Note that the spatial arrangement of voxels creating a homogenization domain is governed by the multinomial distribution with 3 distinct "experiments" (Feller, 2008), i.e., voxels corresponding to the solid phase, water and organic matter. As a result, the value of thermal conductivity of a randomly chosen voxel is independent of all other voxels. Such procedure creates, in some sense, a random field of the thermal conductivity coefficient, with the fluctuation scale converging towards zero (see, e.g., Vanmarcke, 1977; Torquato, 2013; Puła and 

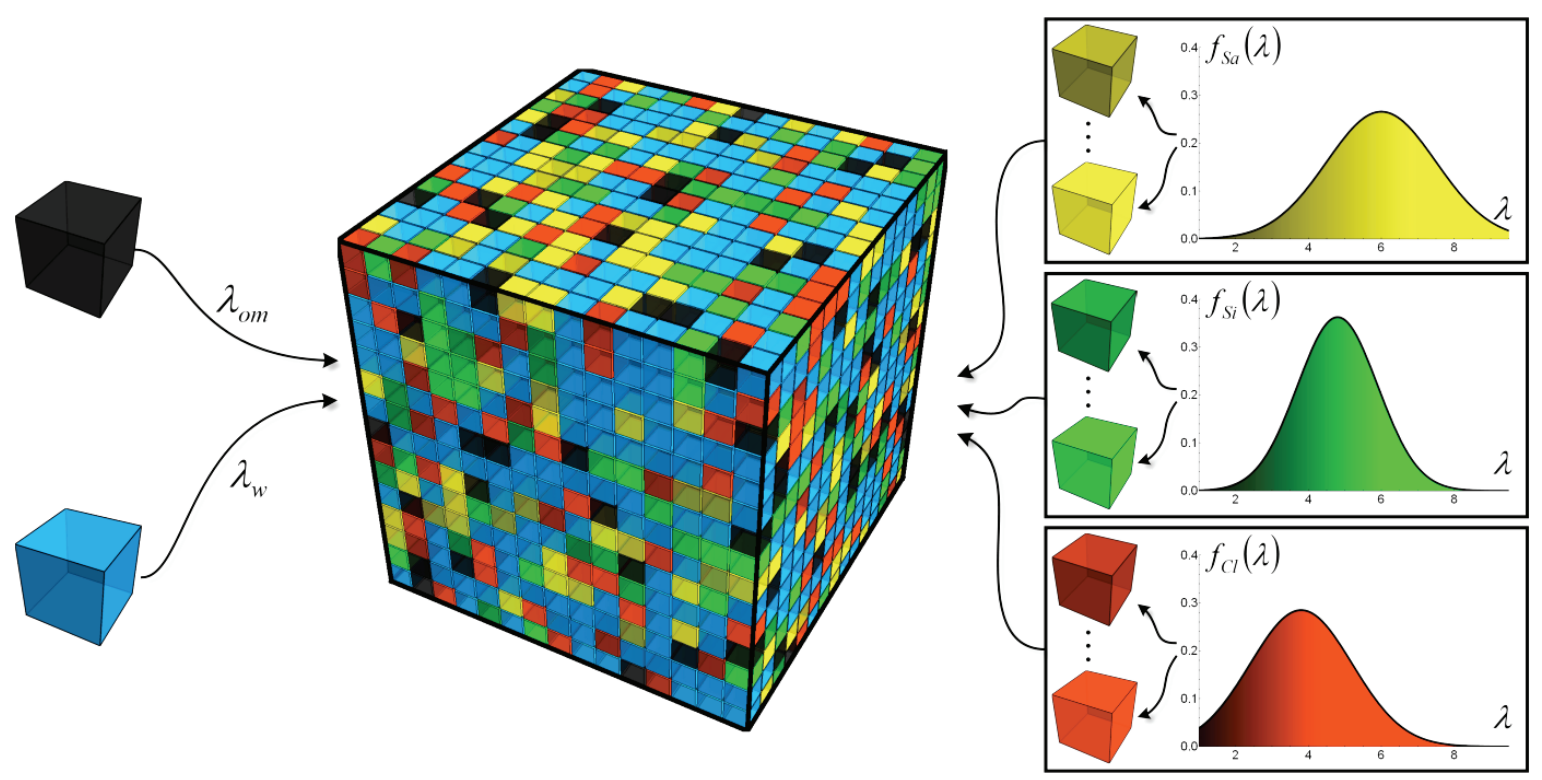

Fig. 1. Graphical presentation of the homogenization domain

Chwała, 2015). Figure 1 graphically illustrates the methodology used for the generation of homogenization domain geometry.

Evaluation of the homogenized thermal conductivity requires a solution of the heat flow boundary value problem stated for the Representative Volume Element (RVE) being a statistically representative realization, say $\omega_{j}$, of the homogenization domain shown in Fig. 1 (Kanit et al. 2003; Łydżba and Różański, 2014). The constituents of the medium (voxels) are isotropic. So, the appropriate boundary value problem for the determination of homogenized soil thermal conductivity is as follows

$$
\left\{\begin{array}{c}
\frac{\partial}{\partial x_{i}} q_{i}^{m}=0, \quad \text { in } V_{\mathrm{RVE}} \\
q_{i}^{m}=-\lambda\left(\mathbf{x}, \omega_{j}\right) \frac{\partial T^{m}}{\partial x_{i}}, \quad \text { in } V_{\mathrm{RVE}} \\
T^{m}\left(\mathbf{x}, \omega_{j}\right)=\delta_{m} x_{i}+\vartheta^{m}\left(\mathbf{x}, \omega_{j}\right), \quad \text { in } V_{\mathrm{RVE}} \\
\vartheta^{m} \text { - periodic, } q_{i}^{m} t_{i}-\text { antiperiodic on } \partial V_{\mathrm{RVE}}
\end{array}\right.
$$

where $q_{i}^{m}$ is the $i$-th component of the heat flux vector, $T^{m}$ is the temperature field, $\vartheta^{m}(\mathbf{x})$ is the socalled corrector field, $\lambda(\mathbf{x})$ is the thermal conductivity coefficient, $\delta_{i j}$ is the Kronecker delta, and $t_{i}$ is the component of a unit normal vector. The superscript $m$ means that the boundary value problem corresponds to the unit macroscopic temperature gradient acting in the direction $x_{m}$. Prescribing periodic boundary conditions implies that $\vartheta^{n}$ takes the same values at two homologous points on opposite faces of RVE, whereas the flux $q_{i}^{m} t_{i}$ takes the opposite values at these points. The boundary value problem (3) admits a unique solution up to the constant value of the temperature. Assuming macroscopic isotropy of the medium considered, the homogenized thermal conductivity, for $\omega_{j}$ realization, can be calculated using a simplified form

$$
\left\langle\lambda\left(\omega_{j}\right)\right\rangle=\frac{1}{3}\left(\frac{1}{V_{\mathrm{RVE}}} \int_{V_{\mathrm{RVE}}}\left(q_{1}^{1}+q_{2}^{2}+q_{3}^{3}\right) d \mathbf{x}\right)
$$

where $\langle$.$\rangle is the volume averaging operator.$

One has to be aware that since it is computationally infeasible to cover the entire realization space, the homogenized thermal conductivity is sampled in a Monte Carlo sense (Gusev, 1997). In other words, determination of the homogenized thermal conductivity requires the solution of the sufficient number of boundary value problem (10), each stated separately for distinct $\omega_{j}$ realization of RVE. Finally, the soil thermal conductivity is evaluated as the Monte Carlo estimator

$$
\lambda^{\mathrm{hom}}=\frac{1}{N} \sum_{j=1}^{N}\left\langle\lambda\left(\omega_{j}\right)\right\rangle
$$

where $N$ is the sufficient number of realizations usually estimated based on the Central Limit Theorem (Feller, 2008). 


\section{RECOVERY PROCEDURE}

The recovery procedure consists in finding such pdfs $f_{C l}(\lambda), f_{S i}(\lambda)$ and $f_{S a}(\lambda)$ for which the homogenized conductivities (equation (5)) match best the laboratory data. The stochastic optimization procedure, namely simulated annealing approach (Kirkpatrick et al., 1983; Černý, 1985) is used for the solution of this inverse problem. Generally, the procedure is as follows: starting from some initial realization of pdfs $f_{i}(\lambda)$, these functions are then evolved towards optimal solutions by minimizing the energy $E$, which at any step, is defined as

$$
E=\frac{1}{N} \sum_{i=1}^{n}\left(\lambda_{i}^{\mathrm{hom}}-\lambda_{i}^{m}\right)^{2}
$$

where $\lambda^{\text {hom }}$ and $\lambda^{m}$ denote the numerical prediction (equation (5)) and measured value of soil thermal conductivity, respectively and $n$ is the number of laboratory measurements taken into consideration. At any step, the configuration of the chosen pdf $f_{i}(\lambda)$ is changed causing the change in the energy function such that $E \rightarrow E^{*}$. Therefore, the difference between two successive states can be calculated, i.e.,

$$
\Delta E=E^{*}-E .
$$

Then, the change of the pdf $f_{i}(\lambda)$ is accepted with certain probability $P$ and is performed in accordance to the Metropolis algorithm (Metropolis et al., 1953)

$$
P\{\Delta E\}=\left\{\begin{array}{cc}
1, & \Delta E \leq 0 \\
\exp \left(-\frac{\Delta E}{T}\right), & \Delta E>0
\end{array}\right.
$$

where $T$ is the fictitious temperature (control parameter).

Usually, the simulated annealing approach requires a large number of iterations until the procedure is stopped. As a consequence, the boundary value problem (3), whose result is involved in equation (6), has to be solved many times. It triggers that the procedure could be cumbersome and time consuming. In order to improve the efficiency of the method we propose to use "approximate" solution of the boundary value problem (3), say $\overline{\lambda^{\text {hom }}}$, which is based on the geometric mean equation. Preliminary simulations showed that, for a two-phase medium with deterministic conductivities, the solution of the boundary value problem (3) almost coincides with the value obtained with the use of geo- metric mean equation (1). In Fig. 2, a numerical solution (homogenized conductivity, equation (5)) as well as the geometric mean value (1) are plotted against the volume fraction of one of the constituents $\phi$.

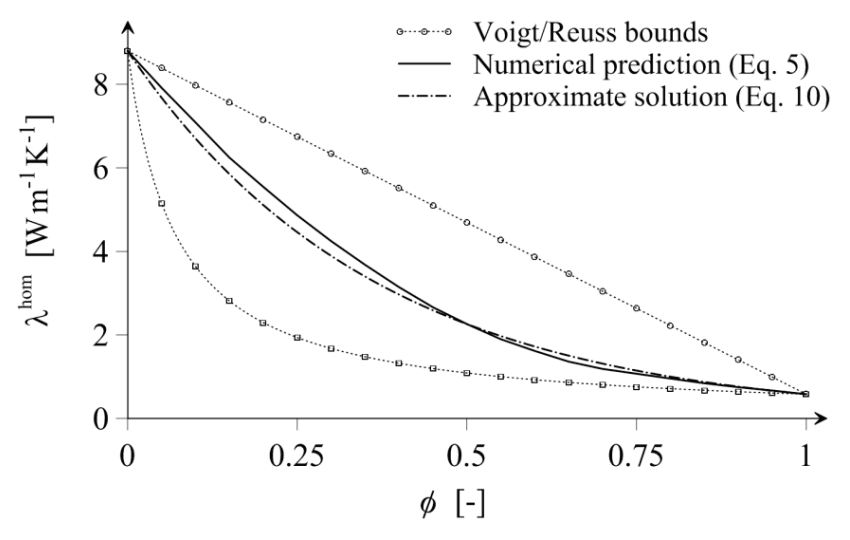

Fig. 2. Numerical solution (equation (5)) and the geometric mean approximation (equation (1)) for a two-phase medium with deterministic conductivities plotted against the volume fraction of one of the constituents

It can be seen that both results are identical when the volume fraction of one of the constituents is approximately equal to 0.5 . For other values of $\phi$ both solutions only slightly differ from each other. We expect the same results for the case of multiphase random medium, so it is proposed to use the geometric mean result instead of numerical solution whenever the energy (6) is calculated. The validity of this "working" hypothesis for the general case, random multiphase medium, is numerically tested in Section 5, where the overall thermal conductivity according to boundary value problem (3) is compared with estimation based on the geometric mean.

Within the numerical procedure, each continuous function $f_{i}(\lambda)$ is approximated by the discrete distribution, so the interval $\left[\lambda_{\min }, \lambda_{\max }\right]$ is discretized in $N_{\lambda}$ subintervals of equal lengths $\Delta$, each with the assigned discrete probability value $f_{i}(\lambda) \cdot \Delta$. At the beginning of the process, for each soil separate, we assume the initial configuration of $f_{i}(\lambda) \cdot \Delta$ in the form of uniform distribution and then the system is evolved towards the optimized solution by minimizing the energy $E$, whose value is obtained with the use of approximate solution $\overline{\lambda^{\text {hom }}}$, i.e.,

$$
E=\frac{1}{n} \sum_{i=1}^{n}\left(\overline{\lambda^{\text {hom }}}-\lambda_{i}^{m}\right)^{2}
$$

where $\overline{\lambda^{\text {hom }}}$ is the approximation of $\lambda^{\text {hom }}$ expressed in the form of the geometric mean equation 


$$
\overline{\lambda^{\mathrm{hom}}}=\left(\left(\left(\prod_{i} \lambda_{i}^{f_{C l}\left(\lambda_{i}\right) \cdot \Delta}\right)^{\phi_{C l}} \cdot\left(\prod_{i} \lambda_{i}^{f_{S i}\left(\lambda_{i}\right) \cdot \Delta}\right)^{\phi_{S i}} \cdot\left(\prod_{i} \lambda_{i}^{f_{S a}\left(\lambda_{i}\right) \cdot \Delta}\right)^{\phi_{S a}}\right)^{1-\phi_{o m}} \cdot \lambda_{o m}^{\phi_{o m}}\right)^{1-\phi_{w}} \cdot \lambda_{w}^{\phi_{w}} \cdot
$$

In the equation above, $\phi_{C l}, \phi_{S i}, \phi_{S a}$ denote the volume fractions of soil separates, i.e., clay, silt and sand, respectively, and $\lambda_{i}$ is the discrete value of the thermal conductivity coefficient. Note that, using equation (10) (instead of numerical solution (5)) in the calculation of energy $E$ substantially decreases the computation time resulting in the high computational efficiency of the proposed approach.

To evolve the system, in particular, to minimize the energy (9), first we randomly choose the soil separate - sand, silt or clay - which is going to be modified at particular step. Next, we draw two discrete values, say $\lambda_{p}$ and $\lambda_{q}$ (such that $p \neq q$ ) and the discrete probabilities of selected $\lambda$, i.e. $f_{i}\left(\lambda_{p}\right) \cdot \Delta$ and $f_{i}\left(\lambda_{q}\right) \cdot \Delta$ are modified. The modification consists in the increase of discrete probability corresponding to one of them and simultaneously the decrease of the second one, by the same constant value 0.005 of the initial probability. The decision whose probability (for $\lambda_{p}$ or $\lambda_{q}$ ) is increased or decreased is evaluated with $50 \%$ probability. The change of probabilities is accepted following the rule given by equation (8). The actual value of the temperature is defined by the cooling schedule and is usually performed as a geometric sequence $T^{j}=\alpha T^{j-1}$ with $\alpha<1$ (Kirkpatrick et al., 1983); $T^{j-1}$ and $T^{j}$ are consecutive temperature values of the cooling steps. The initial value and the rate of change of $T$ is referred to as the cooling schedule, and generally, it is governed by the well-known fact, i.e., a system heated to a high temperature and then slowly cooled down to absolute zero equilibrates to its ground state (Torquato, 2013). The annealing procedure is stopped when after few consecutive temperature loops " $j$ ", the accept rate is less than assumed - relatively low - value.

\section{RESULTS AND DISCUSSION}

In order to recover pdfs $f_{i}(\lambda)$, with the use of previously proposed procedure, a group of soils at saturation is used. Fine grained soils (soils no. 1-17) are studied and the necessary physical parameters of the soils were obtained by the authors. The measurements were performed in the laboratory of Geotechnics and Hydrotechnics Department of Wrocław University of Technology. In order to consider a wide range of soil textures, the data is supplemented by the literature results: soils no. 18-28 from Lu et al. (2007), soil no. 29 from $\mathrm{Lu}$ et al. (2011), soil no. 30 from $\mathrm{Lu}$ et al. (2013), soils no. 31-32 from Ochsner et al. (2001). Fabric composition, porosity, the organic matter content as well as measured thermal conductivities $\left(\lambda^{m}\right)$ of investigated soils are listed in Table 1.

Recovery procedure, presented in the previous Section, is applied to the results of laboratory measurements - thermal conductivities at saturation, $\lambda^{m}$. Each pdf $f_{i}(\lambda)$ is double bounded by the minimum $\left(\lambda_{\min }\right)$ and maximum $\left(\lambda_{\max }\right)$ values, i.e., 2 and $8.8 \mathrm{~W} \mathrm{~m}^{-1} \mathrm{~K}^{-1}$, respectively. These values are assumed based on the ranges of variation as well as characteristic values of thermal conductivities of forming minerals found in the works of Farouki (1981), Clauser and Huenges (1995), Coté and Kon$\operatorname{rad}(2005 \mathrm{~b})$. The number of subintervals, discretizing the range of $\lambda$, is $N_{\lambda}=40$. The initial value of temperature $T_{0}$ is evaluated by numerical testing to give acceptance rate (the fraction of accepted changes) of 0.5 . The annealing scheme as $T^{j}=0.9 T^{j-1}$ is applied. At each temperature value 1,000 changes of discrete probabilities are carried out. The annealing procedure is stopped when after a few consecutive changes of temperature the acceptance rate is less than $0.5 \%$.

Figure 3 visualizes the evolution of probability density functions $f_{i}(\lambda)$ corresponding to particular soil separates. Symbol " $j$ " represents the numbering of temperature loops, i.e., $j=0$ is the initial configuration (uniform distribution), $j=10$ and $j=20$ are some (chosen) intermediate states, and $j=50$ is the final state - the configuration of pdfs which stops the optimization procedure; so, the minimum of $E$ (equation (9)) is obtained after 50,000 iterations. Observing the results we can see that the pdfs for clay and silt separates, $f_{C l}(\lambda)$ and $f_{S i}(\lambda)$, evolve towards triangular distributions whereas $f_{S a}(\lambda)$ converges towards the trapezoidal one. Note that both the pdfs $f_{C l}(\lambda)$ and $f_{S i}(\lambda)$ concentrate around lower bound of $\lambda$, while $f_{S a}(\lambda)$ exhibits opposite behaviour. This result is in accordance with the commonly known fact - thermal conductivity of quartz minerals (which is mainly forming sand grains) is approximately $2-3$ times higher than the clay or silt ones. 
Table 1. Properties of soils under investigation

\begin{tabular}{|c|c|c|c|c|c|c|}
\hline \multirow{3}{*}{$i$} & \multicolumn{3}{|c|}{ Fabric composition } & \multirow{2}{*}{$\phi_{w}$} & \multirow{2}{*}{$\phi_{o m}$} & \multirow{2}{*}{$\lambda^{m}$} \\
\hline & $\phi_{C l}$ & $\phi_{S i}$ & $\phi_{S a}$ & & & \\
\hline & {$[\%]$} & {$[\%]$} & [\%] & {$[-]$} & {$[\%]$} & {$\left[\mathrm{W} \mathrm{m}^{-1} \mathrm{~K}^{-1}\right]$} \\
\hline 1 & 36 & 58 & 6 & 0.36 & 0.25 & 1.90 \\
\hline 2 & 43 & 45 & 12 & 0.24 & 0.42 & 2.51 \\
\hline 3 & 39 & 49 & 12 & 0.29 & 4.52 & 1.90 \\
\hline 4 & 54 & 46 & 0 & 0.37 & 2.15 & 1.64 \\
\hline 5 & 36 & 58 & 6 & 0.42 & 3.62 & 1.49 \\
\hline 6 & 70 & 24 & 6 & 0.38 & 0.18 & 1.78 \\
\hline 7 & 58 & 37 & 5 & 0.35 & 0.35 & 1.94 \\
\hline 8 & 41 & 57 & 2 & 0.31 & 0.05 & 2.23 \\
\hline 9 & 46 & 50 & 4 & 0.26 & 1.20 & 2.10 \\
\hline 10 & 41 & 32 & 27 & 0.34 & 0.08 & 2.33 \\
\hline 11 & 58 & 37 & 5 & 0.42 & 0.38 & 1.72 \\
\hline 12 & 7 & 92 & 1 & 0.43 & 0.05 & 1.96 \\
\hline 13 & 36 & 28 & 36 & 0.27 & 0.23 & 2.61 \\
\hline 14 & 54 & 42 & 4 & 0.27 & 1.65 & 2.10 \\
\hline 15 & 42 & 57 & 1 & 0.50 & 0.10 & 1.43 \\
\hline 16 & 60 & 33 & 7 & 0.36 & 0.05 & 2.15 \\
\hline 17 & 39 & 40 & 21 & 0.29 & 0.65 & 2.55 \\
\hline 18 & 5 & 1 & 94 & 0.40 & 0.09 & 2.32 \\
\hline 19 & 12 & 21 & 67 & 0.48 & 0.86 & 1.75 \\
\hline 20 & 11 & 49 & 40 & 0.51 & 0.49 & 1.58 \\
\hline 21 & 22 & 51 & 27 & 0.50 & 1.19 & 1.38 \\
\hline 22 & 27 & 54 & 19 & 0.51 & 0.39 & 1.33 \\
\hline 23 & 32 & 60 & 8 & 0.51 & 3.02 & 1.29 \\
\hline 24 & 30 & 38 & 32 & 0.51 & 0.27 & 1.35 \\
\hline 25 & 6 & 1 & 93 & 0.40 & 0.07 & 2.05 \\
\hline 26 & 1 & 7 & 92 & 0.40 & 0.60 & 2.09 \\
\hline 27 & 9 & 41 & 50 & 0.48 & 0.25 & 1.72 \\
\hline 28 & 19 & 70 & 11 & 0.51 & 0.84 & 1.60 \\
\hline 29 & 43 & 50 & 7 & 0.51 & 2.09 & 1.24 \\
\hline 30 & 5 & 1 & 94 & 0.43 & 0.09 & 1.86 \\
\hline 31 & 13 & 64 & 23 & 0.56 & 0.90 & 1.21 \\
\hline 32 & 32 & 56 & 12 & 0.53 & 1.10 & 1.38 \\
\hline
\end{tabular}

Continuous functions are then fitted to the final configurations of discrete pdfs obtained with simulated annealing approach. Graphical presentation of continuous pdfs for particular soil separates, namely clay, silt and sand is provided in Fig. 4.

Mathematical formulas of probability density functions $f_{i}(\lambda)$ are as follows

$f_{C l}(\lambda)=\left\{\begin{array}{cc}-5.945 \cdot 10^{-2} \cdot \lambda+4.637 \cdot 10^{-1}, & 2 \leq \lambda \leq 7.8, \\ 0, & \text { otherwise }\end{array}\right.$

$f_{S i}(\lambda)=\left\{\begin{array}{cc}-4.325 \cdot 10^{-2} \cdot \lambda+3.806 \cdot 10^{-1}, & 2 \leq \lambda \leq 8.8, \\ 0, & \text { otherwise }\end{array}\right.$

$$
f_{S a}(\lambda)=\left\{\begin{array}{cc}
1.136 \cdot 10^{-2} \cdot \lambda+8.727 \cdot 10^{-2}, & 2 \leq \lambda \leq 8.8 \\
0, & \text { otherwise }
\end{array}\right.
$$

Now, we verify the correctness of the recovered probability density functions $f_{i}(\lambda)$. In other words, identified functions (equations (11)-(13)) are now applied to the computational micromechanics approach (described in Section 3) and the predictions of overall thermal conductivities at saturation (of all soils considered) are evaluated. In Fig. 5a, a numerical prediction of thermal conductivity $\lambda^{\text {hom }}$ (equation (5)) is plotted against the approximate solution $\overline{\lambda^{\text {hom }}}$ (Eq. 10) - the one used in the recovery procedure for the purpose of computational efficiency. 

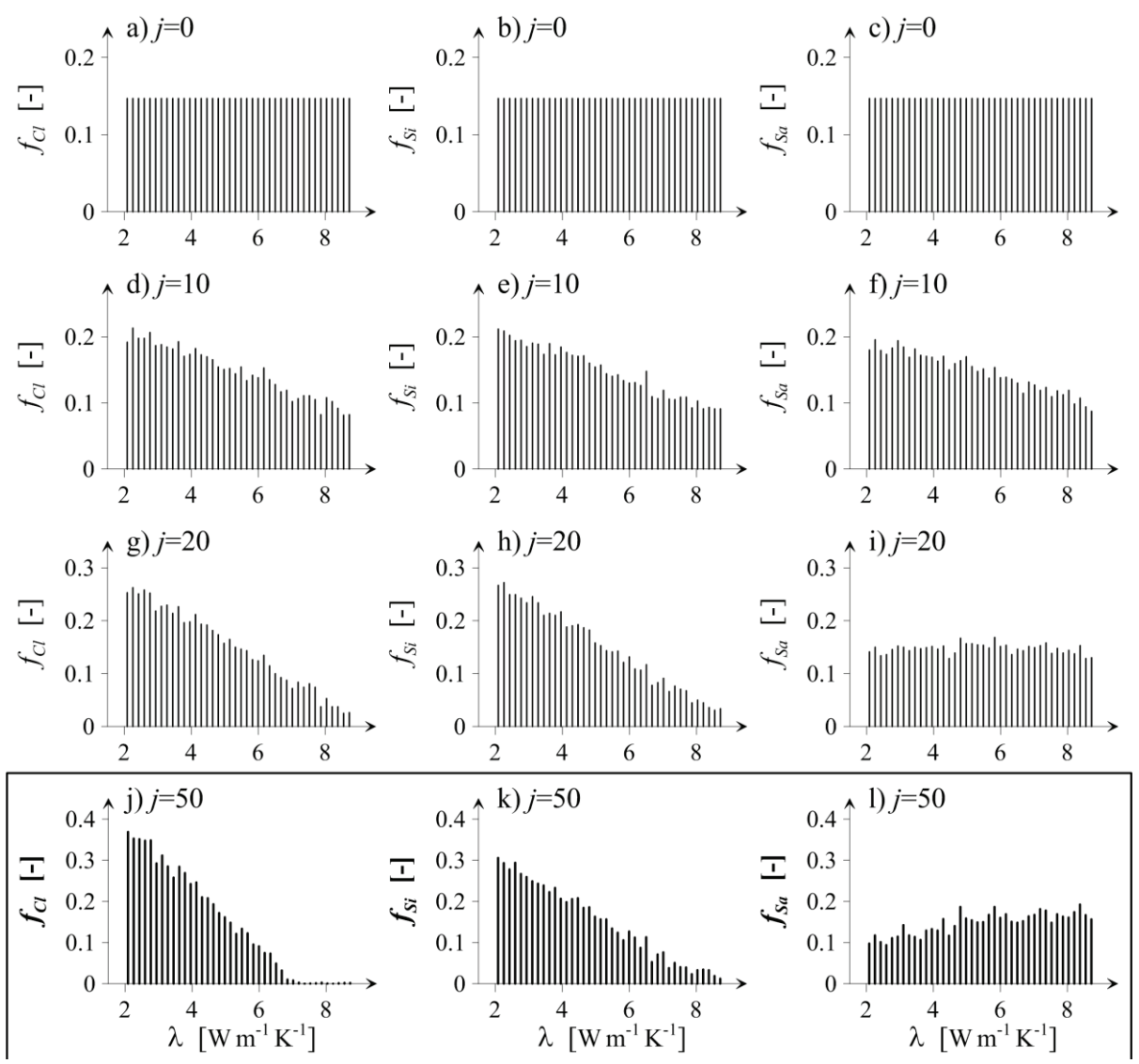

Fig. 3. The evolution of discrete probability density functions $f_{i}(\lambda)$ for particular soil separates: clay, silt and sand
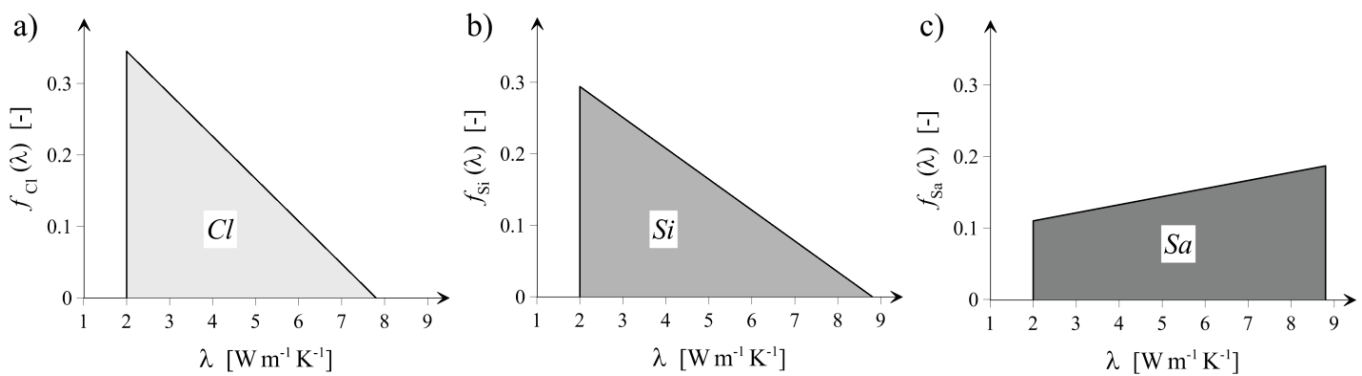

Fig. 4. Recovered continuous probability density functions $f_{i}(\lambda)$ for particular soil separates: clay, silt and sand
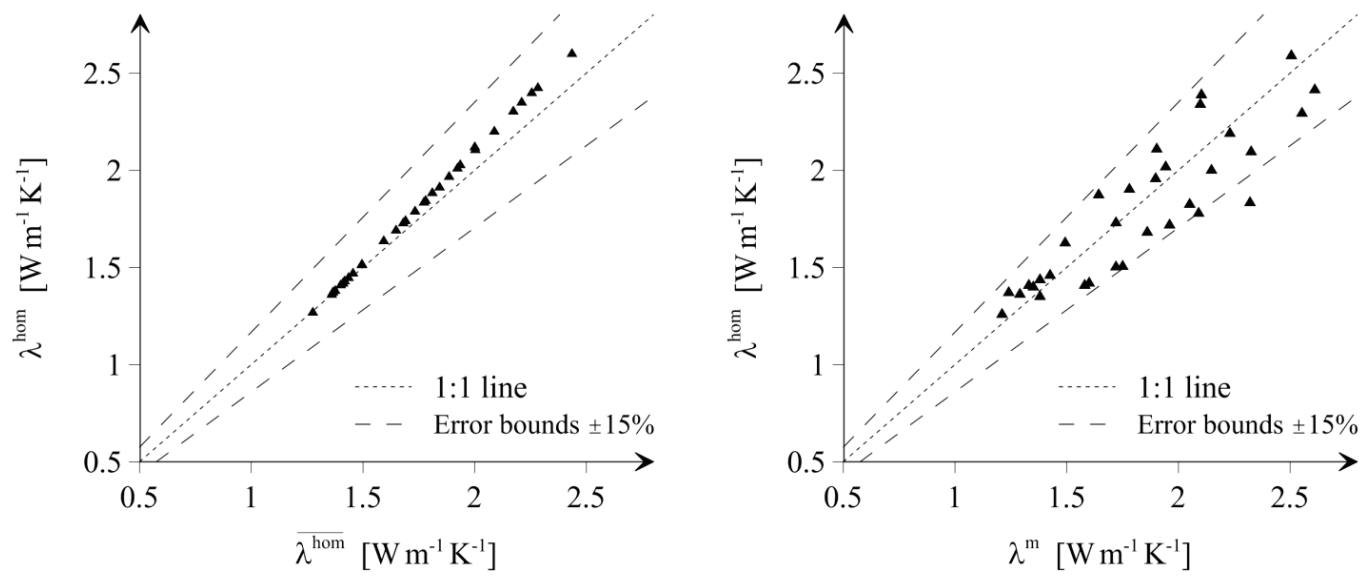

Fig. 5. Numerical prediction of soil thermal conductivity (equation (5)) vs.:

(a) the approximate solution (equation (10)); (b) measured thermal conductivity at saturation 
It can be seen that both results are in a good agreement; numerical solution only slightly overestimates the approximate one. So, the postulate of utilizing $\overline{\lambda^{\text {hom }}}$ instead of $\lambda^{\text {hom }}$ in the formulation of energy $E$ is correct; geometric mean equation (10) is the approximate solution of $\lambda^{\text {hom }}$ for the multiphase medium considered. In Fig. 5 b, the numerical predictions of soils thermal conductivities $\left(\lambda^{\text {hom }}\right)$ are plotted vs. the measured ones $\left(\lambda^{m}\right)$. For comparison purposes 1:1 line is also provided. We observe that the predictions almost coincide with laboratory data; the results are within $15 \%$ error bounds. Obtained results confirm the effectiveness of the proposed recovery procedure - identified $\operatorname{pdfs} f_{i}(\lambda)$, when applied to the computational micromechanics approach, ensure good agreement of predicted and measured thermal conductivities.

\section{FINAL CONCLUSIONS}

In the study, we propose to model the complex microstructure of the soil solid by prescribing the spatial variability of thermal conductivity coefficient to distinct soil separates, namely clay, silt and sand. For that purpose, we postulate that the variation of thermal conductivity coefficient of each soil separate " $i$ " can be represented by some probability density function $f_{i}(\lambda)$. The main goal of the work was the recovery of these pdfs $f_{i}(\lambda)$ with the use of micromechanics-based back analysis. In other words, we aimed to find such pdfs $f_{C l}(\lambda), f_{S i}(\lambda), f_{S a}(\lambda)$ that ensure good agreement between numerically predicted and measured overall soil thermal conductivities at saturation. As a procedure of the back analysis we use the stochastic optimization approach, i.e., the simulated annealing algorithm.

In the following, we formulate main conclusions that can be drawn from the present study:

- the algorithm based on the simulated annealing approach can be successfully used for recovery/ identification of $\operatorname{pdfs} f_{i}(\lambda)$,

- since the procedure requires approximately 50,000 iterations to find the optimized solution, computation times - needed to find the optimized solution - are extremely large; this is caused by the formulation of energy (equation (7)) which involves $\lambda^{\text {hom }}$,

- based on independent tests, performed for a twophase random media with deterministic conductivities, we showed that $\lambda^{\text {hom }}$ can be approximated by the geometric mean value $\overline{\lambda^{\text {hom }}}$, equation (10),
- using approximate solution $\overline{\lambda^{\text {hom }}}$ (instead of $\lambda^{\text {hom }}$ ) in the calculation of energy $E$ makes the proposed recovery procedure computationally efficient,

- recovered pdfs for clay and silt separates, i.e., $f_{C l}(\lambda)$ and $f_{S i}(\lambda)$, take the form of triangular distributions and they both concentrate around minimum value of $\lambda$,

- recovered pdf for sand separate, i.e., $f_{S a}(\lambda)$, takes the form of the trapezoidal distribution and it mainly concentrates around maximum value of $\lambda$,

- applying recovered pdfs $f_{i}(\lambda)$ to the computational micromechanics approach results in a good agreement between predicted and measured thermal conductivities of soils at saturation.

\section{REFERENCES}

[1] BRIstow K.L., Thermal conductivity, [in:] Methods of Soil Analysis. Part 4. Physical methods, Soil Science Society of America Book Ser. 5, SSSA and ASA, Madison, WI, 2002, 1209-1226.

[2] ČERNÝ V., Thermodynamical approach to the traveling salesman problem: An efficient simulation algorithm, Journal of optimization theory and applications, 1985, 45(1), 41-51.

[3] Clauser C., Huenges E., Thermal conductivity of rocks and minerals, Rock physics Phase Relations: A handbook of physical constants, 1995, 105-126.

[4] Côté J., KonRad J.M., Thermal conductivity of base-course materials, Canadian Geotechnical Journal, 2005a, 42(1), 61-78.

[5] CôtÉ J., KonRAD J.M., A generalized thermal conductivity model for soils and construction materials, Canadian Geotechnical Journal, 2005b, 42(2), 443-458.

[6] FAROUKI O.T., Thermal properties of soils (CRREL Monogr. 81-1). United States Army Corps of Engineers, Cold Regions Research and Engineering Laboratory, Hanover, New Hampshire, 1981.

[7] Feller W., An Introduction to Probability Theory and Its Applications, John Wiley \& Sons, Vol. 2, 2008.

[8] GEMANT A., How to compute thermal soil conductivities, Heating, Piping and Air Conditioning, 1952, 24(1), 122-123.

[9] Gusev A.A., Representative volume element size for elastic composites: a numerical study, Journal of the Mechanics and Physics of Solids, 1997, 45(9), 1449-1459.

[10] Johansen O., Thermal conductivity of soils, Ph.D. dissertation, Norwegian University of Science and Technology, Trondheim (CRREL draft translation 637, 1977), 1975.

[11] Kanit T., Forest S., Galliet I., Mounoury V., Jeulin D., Determination of the size of the representative volume element for random composites: statistical and numerical approach, International Journal of Solids and Structures, 2003, 40(13), 3647-3679.

[12] KirkPatrick S., Gelatt C.D., Vecchi M.P., Optimization by simulated annealing, Science, 1983, 220(4598), 671-680.

[13] Lu S., REN T., GONG Y., HoRTON R., An improved model for predicting soil thermal conductivity from water content at room temperature, Soil Science Society of America Journal, 2007, 71(1), 8-14.

[14] Lu S., Ren T., Yu Z., Horton R., A method to estimate water vapour enhancement factor in soil, European Journal of Soil Science, 2011, 62(4), 498-504. 
[15] Lu Y., Lu S., Horton R., Ren T., An empirical model for estimating soil thermal conductivity from texture, water content, and bulk density, Soil Science Society of America Journal, 2014, 78(6), 1859-1868.

[16] ŁyDŻBA D., RóŻAŃSKi A., Microstructure measures and the minimum size of a representative volume element: $2 D \mathrm{nu}$ merical study, Acta Geophysica, 2014, 62(5), 1060-1086.

[17] OchSner T.E., Horton R., Ren T., A new perspective on soil thermal properties, Soil Science Society of America Journal, 2001, 65(6), 1641-1647.
[18] PuŁa W., ChwaŁa M., On spatial averaging along random slip lines in the reliability computations of shallow strip foundations, Computers and Geotechnics, 2015, 68, 128-136.

[19] TORQUATO S., Random heterogeneous materials: microstructure and macroscopic properties, Springer Science and Business Media, 2013, 16.

[20] Vanmarcke E.H., Probabilistic modeling of soil profiles, Journal of the Geotechnical Engineering Division, 1977, 103(11), 1227-1246. 